\title{
Guía de manejo Hiperplasia Prostática Benigna (SCU 2021)
}

\section{Benign Prostatic Hyperplasia Guideline (SCU 2021)}

\author{
Hugo López-Ramos ${ }^{1}$ Carlos Latorre ${ }^{2}$ Germán Patiño $^{3} \quad$ Juliana Arenas ${ }^{4}$
}

1 Profesor y Jefe del Programa de Urología. Pontificia Universidad Javeriana. Hospital Universitario San Ignacio. Bogotá, Colombia

2 Urólogo. Clínica del Country. Bogotá, Colombia

3 Urólogo. Unidad de Urología Reconstructiva. Hospital Universitario San Ignacio. Bogotí, Colombia

${ }^{4}$ Departamento de Urologia. Pontificia Universidad Javeriana.

Hospital Universitario San Ignacio. Bogotá, Colombia

Urol Colomb 2021;30:145-152.
Dirección de correspondencia Hugo López-Ramos, Carrera 7 \# 40-62, Bogotá, Colombia (e-mail: lopez.he@javeriana.edu.co).

atención adecuada. Dicha evaluación incluye historia clínica completa y un examen físico enfocado.

Enfatizamos que la correlación del tacto rectal con el volumen es pobre y existe la posibilidad de subestimar el volumen prostático cuando la próstata es $>30 \mathrm{cc}$.

Entre los exámenes complementarios se incluyen el uroanálisis, función renal, evaluación de la glicemia, diario miccional. Se recomienda la realización del PSA en hombres mayores de 40 años con una expectativa de vida mayor a 10 años. Se deberá realizar una ecografía de vías urinarias, para medición del volumen prostático, evaluación de complicaciones en el tracto urinario superior, y la medición del residuo postmiccional (RPM). Aunque no es mandatoria en la evaluación diagnóstica, el panel de la guía la recomienda como opcional. Se recomienda una uroflujometría, para evaluar la respuesta al tratamiento médico o quirúrgico y en la determinación de la correlación de los síntomas con hallazgos objetivos. La cistoscopia está indicada en el estudio de los pacientes con hematuria micro o macroscópica, estrechez uretral o cáncer de vejiga concomitantes con SOUB.

Los estudios de flujo-presión están indicados en pacientes en quienes los síntomas generen duda en el diagnóstico, en pacientes con enfermedad neurológica, quienes hayan tenido cirugía pélvica radical previa, radioterapia pélvica, diabetes no controlada y pacientes con $\mathrm{RPM}>300 \mathrm{~mL}$, así como aquellos que hayan tenido procedimientos quirúrgicos previos fallidos.
DOI https://doi.org/ 10.1055/s-0041-1731669. ISSN 0120-789X. e ISSN 2027-0119.
(C) 2021. Sociedad Colombiana de Urología. All rights reserved. This is an open access article published by Thieme under the terms of the Creative Commons Attribution-NonDerivative-NonCommercial-License, permitting copying and reproduction so long as the original work is given appropriate credit. Contents may not be used for commercial purposes, or adapted, remixed, transformed or built upon. (https://creativecommons.org/ licenses/by-nc-nd/4.0/)

Thieme Revinter Publicações Ltda., Rua do Matoso 170, Rio de Janeiro, RJ, CEP 20270-135, Brazil 
No está indicado el uso de ecografía transrectal de forma rutinaria en la evaluación de los SOUB secundarios a crecimiento prostático benigno.

\section{MANEJO DE LA ENFERMEDAD}

\subsection{Manejo Conservador}

Los hombres con SOUB no complicados, leves o moderados, podrán ser candidatos a manejo expectante. La evaluación periódica de los pacientes en protocolo de vigilancia activa incluye la medición del RPM, la evaluación de la severidad de los síntomas, con una educación sobre la condición al paciente, descartando causa oncológica y brindando asesoría sobre los hábitos de vida saludable, cada 3, 6 y 12 meses y posteriormente de forma anual si no hay cambios. ${ }^{10}$ Se deberá asesorar en hábitos de estilo de vida saludable. La tasa de fracaso del tratamiento durante un período de cinco años fue del $21 \%{ }^{11}$ Nivel de evidencia $1 \mathrm{~b}$.

\subsection{Tratamiento farmacológico}

\subsubsection{Antagonistas de los alfa-1-adrenorreceptores} Los bloqueadores $\alpha 1$ generalmente se consideran el tratamiento farmacológico de primera línea para los SUOB moderados debido a su inicio de acción rápida, buena eficacia y baja prevalencia y gravedad de los eventos adversos. Las comparaciones indirectas y directas entre los bloqueadores $\alpha 1$ demuestran que todos los bloqueadores $\alpha 1$ tienen una eficacia similar en dosis apropiadas ${ }^{12}$ con disminución del IPSS en un 30 a $40 \%$ e incrementan el $Q_{\text {máx }}$ aproximadamente en un $20-25 \%$. Hasta la fecha, se han realizado al menos 15 revisiones sistemáticas de los bloqueadores $\alpha$ en la literatura, todas demostrando que son superiores al placebo en la mejoría de los síntomas. ${ }^{13,14}$ Los efectos vasodilatadores son más pronunciados con doxazosina y terazosina, y son menos comunes con alfuzosina y son tamsulosina. ${ }^{15}$ Un metaanálisis reciente demostró que la disfunción eyaculatoria fue significativamente más común con los bloqueadores $\alpha 1$ que con el placebo (OR 5.88) y se relacionó significativamente con el uso de tamsulosina o silodosina (OR: 8.57 y 32.5). ${ }^{15}$

\subsubsection{Inhibidores de la $5 \alpha$-reductasa}

Los efectos clínicos relativos al placebo se observan después de un tratamiento de al menos seis meses. Después de dos o cuatro años de tratamiento, mejoran el IPSS en aproximadamente un 15-30\%, disminuyen el volumen de la próstata en un $18-28 \%$ y aumentan el $\mathrm{Q}_{\text {máx }}$ en $1.5-2.0 \mathrm{~mL} / \mathrm{s}$ en pacientes con SOUB causados por el crecimiento de la próstata. ${ }^{16}$ Los inhibidores de la $5 \alpha$-reductasa, pero no los bloqueadores $\alpha 1$, reducen el riesgo a largo plazo (más de 1 año) de RUA así como la necesidad de cirugía. ${ }^{17}$ Un análisis agrupado de tres ensayos aleatorizados con datos de seguimiento de dos años, mostró que el tratamiento con finasteride disminuyó el riesgo relativo de RUA en un 57\%, y la intervención quirúrgica en un $34 \%$ en pacientes con SUOB moderados (17). El tratamiento con inhibidores de la $5 \alpha$ reductasa debe considerarse en hombres con SUOB mode- rados a severos y una próstata agrandada $(>40 \mathrm{ml})$ y/o una concentración elevada de PSA ( $>1.4-1.6 \mathrm{ng} / \mathrm{ml}$ ). Pueden prevenir el riesgo de RUA y la necesidad de cirugía. Debido al lento inicio de acción, no son adecuados para uso a corto plazo. Su efecto sobre el PSA debe considerarse en relación con la detección de CaP.

\subsubsection{Antimuscarínicos}

No todos los antimuscarínicos han sido probados en hombres de edad avanzada, los estudios a largo plazo sobre la eficacia de los antagonistas de los receptores muscarínicos en hombres de cualquier edad con SOUB aún no están disponibles. Se recomienda a su uso una reevaluación periódica del IPSS y el RPM. $^{18}$

\subsubsection{Inhibidores de Fosfodiesterasa 5}

Hasta la fecha, solo el tadalafil a dosis de $5 \mathrm{mg}$ una vez al día ha sido autorizado oficialmente para el tratamiento de los SOUB con o sin DE. La experiencia a largo plazo con tadalafil en hombres con SOUB se limita a un ensayo con un seguimiento de un año, por lo tanto, no es posible establecer conclusiones sobre su eficacia o tolerabilidad durante más de un año. ${ }^{19}$

\subsubsection{Fitoterapia}

Sólo se ha recomendado Serenoa repens extraído con hexano (no con etanol) para un uso bien establecido. ${ }^{20}$

\subsubsection{Combinación de Terapias}

4.2.7.1 Bloqueadores $\alpha 1+$ Inhibidores de la $5 \alpha$-reductasa En comparación con los bloqueadores $\alpha 1$ o la monoterapia con un inhibidor de la $5 \alpha$-reductasa, la terapia combinada da como resultado una mejoría más marcada en los SOUB y en el $\mathrm{Q}_{\text {máx }}$ y es superior en la prevención de la progresión de la enfermedad. Sin embargo, la terapia combinada también se asocia con una mayor tasa de eventos adversos. ${ }^{21-23}$ La terapia combinada debe prescribirse principalmente en hombres que tienen SOUB moderados a severos y están en riesgo de progresión de la enfermedad (mayor volumen de próstata, mayor valor de PSA, edad avanzada, mayor RPM, menor $\mathrm{Q}_{\text {máx }}$ ). La terapia combinada solo debe usarse cuando se pretende un tratamiento a largo plazo (más de doce meses) y los pacientes deben ser informados al respecto.

4.2.7.2 Bloqueadores $\alpha 1$ + antagonistas del receptor muscarínico

Los estudios con esta combinación utilizaron principalmente puntos finales de síntomas de almacenamiento, fueron de corta duración e incluyeron solo hombres con bajos volúmenes de RPM al inicio del estudio. Por lo tanto, se recomienda medir el RPM durante el tratamiento combinado.

\subsection{Manejo quirúrgico}

A pesar del advenimiento de las nuevas tecnologías, la resección transuretral de próstata (RTUP) monopolar ha sido la piedra angular del tratamiento quirúrgico para los SOUB por más de nueve décadas. Una amplia investigación clínica buscando una alternativa más efectiva y segura a menudo se ve obstaculizada por limitaciones metodológicas, 
incluyendo un seguimiento inadecuado. Basado en el consenso del Panel, los plazos que definen el seguimiento a corto, mediano y largo plazo de los pacientes sometidos a tratamientos quirúrgicos son 12, 36 y más de 36 meses, respectivamente. Los médicos deben informar a los pacientes que faltan estudios ECAs quirúrgicos a largo plazo.

\subsubsection{Resección transuretral monopolar de la próstata e} incisión transuretral de la próstata.

En un metanálisis que incluía $20 \mathrm{ECAs}$, con un seguimiento máximo de cinco años, la RTUP-M resultó en una mejoría significativa del $Q_{\text {máx }}(+162 \%)$, una reducción significativa en los SOUB (-70\%), una mejoría en el puntaje de la calidad de vida (-69\%) y del RPM (-77\%).

La RTUP-M y la ITUP son tratamientos efectivos para los SOUB moderados a severos secundarios a la obstrucción del tracto urinario inferior. La elección debe basarse principalmente en el volumen de la próstata $(<30 \mathrm{ml}$ y $30-80 \mathrm{ml}$ adecuados para ITUP y RTUP-M, respectivamente). El límite superior para RTUP-M sugerido es de $80 \mathrm{ml}$ (según la opinión del panel de expertos, bajo el supuesto de que este límite depende de la experiencia del cirujano, la elección del tamaño del resectoscopio y la velocidad de resección), a medida que aumenta la duración quirúrgica, hay un aumento significativo en la tasa de complicaciones y el procedimiento es más seguro cuando se realiza en menos de 90 minutos. $^{24,25}$

\subsubsection{Modificaciones de RTUP-M: RTUP bipolar}

La RTUP bipolar en pacientes con SOUB moderados a severos secundarios a obstrucción del tracto inferior, tiene eficacia similar a la RTUP-M, pero con menor morbilidad perioperatoria. La duración de las mejoras con RTUP-B se documentó en varios ECAs con seguimiento a mediano plazo. Los resultados a largo plazo (hasta cinco años) para la RTUP-B han mostrado que la seguridad y la eficacia son comparables con la RTUP-M. La elección de RTUP-B debe basarse sobre la disponibilidad del equipo, la experiencia del cirujano y la preferencia del paciente. ${ }^{26-30}$

\subsection{Modificaciones de la RTUP-B: vaporización transuretral bipolar de la próstata}

La VTUP-B y la RTUP tienen una eficacia similar a corto plazo. La VTUP-B plasmakinética tiene un perfil perioperatorio favorable, una seguridad similar a mediano plazo pero una eficacia inferior a mediano plazo en comparación con la RTUP. La VTUP-B plasma tiene una morbilidad menor a corto plazo en comparación con la RTUP. ${ }^{31,32}$

\subsubsection{Prostatectomía Abierta}

La prostatectomía abierta es el método quirúrgico más invasivo, pero es un procedimiento efectivo y duradero para el tratamiento de los SOUB. En ausencia de un arsenal endourológico que incluya el láser de holmium o un sistema bipolar, la PA es el tratamiento quirúrgico de elección para los hombres con próstata $>80 \mathrm{ml}$.

\subsubsection{Tratamientos con láser}

\subsubsection{Enucleación y resección prostática con laser de Holmium}

La técnica de HoLEP requiere experiencia y habilidades endoscópicas relevantes. La experiencia de la el cirujano fue el factor más importante que afectó la aparición general de complicaciones. ${ }^{33,34}$ Se recomienda una tutoría y asistencia a programas de enseñanza que mejoren el rendimiento de la curva de aprendizaje qurúrgica. ${ }^{34}$ Con la llegada de HoLEP y el hecho de que no hay publicaciones relevantes sobre resección de próstata con láser de Holmium (HoLRP) publicadas desde 2004, la HoLRP de la próstata no juega un papel en el tratamiento contemporáneo de laHPB.

\subsubsection{Vaporización de la próstata con láser de luz verde (Greenlight) de $532 \mathrm{~nm}$}

El láser XPS de 180 W no es inferior a la RTUP en términos de IPSS, $Q_{\text {máx }}$, volumen de RPM, reducción en el volumen prostático, disminución del PSA y en los cuestionarios de calidad de vida. El único ECA disponible para el láser de 180 W mostró resultados de eficacia y seguridad similares a la RTUP con resultados estables a los 24 meses de seguimiento; sin embargo, hubo una tasa de retratamiento más alta después de 24 meses en el brazo de PVP. ${ }^{35}$ De acuerdo al estudio Goliath, la prostatectomía con láser Greenlight de $180 \mathrm{~W}$ no es inferior a la RTUP en términos de complicaciones perioperatorias. La supervivencia libre de reoperación durante 24 meses de seguimiento fue comparable entre el brazo de TURP y el brazo láser XPS $180 \mathrm{~W} .{ }^{35}$ El láser XPS de $180 \mathrm{~W}$ representa el estándar actual de los generadores para PVP; sin embargo, el número y la calidad de las publicaciones de soporte son bajos, especialmente para las próstatas grandes $(>100 \mathrm{ml})$, sin un adecuado seguimiento a largo plazo (evidencia 1b). Se recomienda fuertemente la vaporización de la próstata con láser de potasio-titanil-fosfato (KTP) de $80 \mathrm{~W}$ a $532 \mathrm{~nm}$ a hombres con SOUB moderados a severos con un volumen prostático de $30-80 \mathrm{ml}$ como alternativa a la resección transuretral de la próstata (RTUP). La vaporización de próstata con láser de $120 \mathrm{~W}, 532 \mathrm{~nm}$ y triborato de litio (LBO) a hombres con SOUB moderados a severos con un volumen de próstata de $30-80 \mathrm{ml}$ como alternativa a la RTUP. Se recomienda débilmente la vaporización con láser de la próstata utilizando láser KTP de $80 \mathrm{~W}, 120$ o $180 \mathrm{~W}$ LBO para el tratamiento de pacientes que reciben terapia antiplaquetaria o anticoagulante con un volumen prostático menor a $80 \mathrm{ml}$.

\subsubsection{Tratamiento de la próstata con láser diodo}

La vaporización con láser de diodo conlleva mejoras similares en los parámetros clínicos y sintomáticos durante el seguimiento a corto plazo y proporciona buenas propiedades hemostáticas. La enucleación con láser de diodo (DiLEP) parece ofrecer una eficacia y seguridad similares en comparación con la RTUP o la enucleación bipolar. Recomendación débil, Nivel de evidencia $1 \mathrm{~b}$. 


\subsubsection{Manejo con láser de Thulium:ytrio-aluminio-granate} (Tm: YAG)

Como un número limitado de ECAs y solo pocos estudios con seguimiento a largo plazo apoyan la eficacia de la prostatectomía con láser de tulio, es necesario mantener la investigación continua de la técnica. La enucleación con láser de la próstata mediante vapoenucleación (ThuVEP) o con la técnica (ThuLEP) demuestró una alta seguridad intraoperatoria con respecto a las propiedades hemostáticas en comparación con la RTUP. Los resultados a corto plazo son comparables a la RTUP. Evidencia 1b. Se recomienda débilmente la enucleación con láser de la próstata usando la vapoenucleación Tm: YAG (ThuVEP) y Tm: enucleación anatómica asistida por láser YAG (ThuLEP) a hombres con SOUB moderados a severos como alternativas a la RTUP y a la enucleación con láser de Holmium (HoLEP). ${ }^{36,37}$

\subsubsection{Elevación de la uretra prostática EUP.}

Un lóbulo medio que protruye y genera obstrucción no puede ser tratado de manera efectiva con la elevación prostática, y en próstatas de gran tamaño la efectividad aún no se ha demostrado. Se necesitan estudios a largo plazo para evaluar la duración del efecto de este tipo de tratamiento comparado con otras técnicas. EUP mejora el IPSS, el Qmáx, y la calidad de vida; sin embargo, estas mejorías son inferiores a la RTUP a los 24 meses con evidencia 1 b. ${ }^{38,39}$

\subsubsection{Inyecciones intra prostáticas}

A pesar de que la evidencia experimental para los componentes como la BoNT-A y el PRX302 han sido prometedores para su transición al uso clínico, los resultados positivos de estudios fase II, no se han confirmado en estudios fase III. Se necesitan estudios aleatorizados controlados comparados con tratamientos de referencia para confirmar los resultados prometedores del NX-1207. Se desaconseja el uso de inyección de toxina botulínica-A para el tratamiento de pacientes con SOUB, recomendacion fuerte.

\subsubsection{Técnicas en investigación}

La prostatectomía simple mínimamente invasiva es comparable con la PA en términos de eficacia y seguridad, mostrando

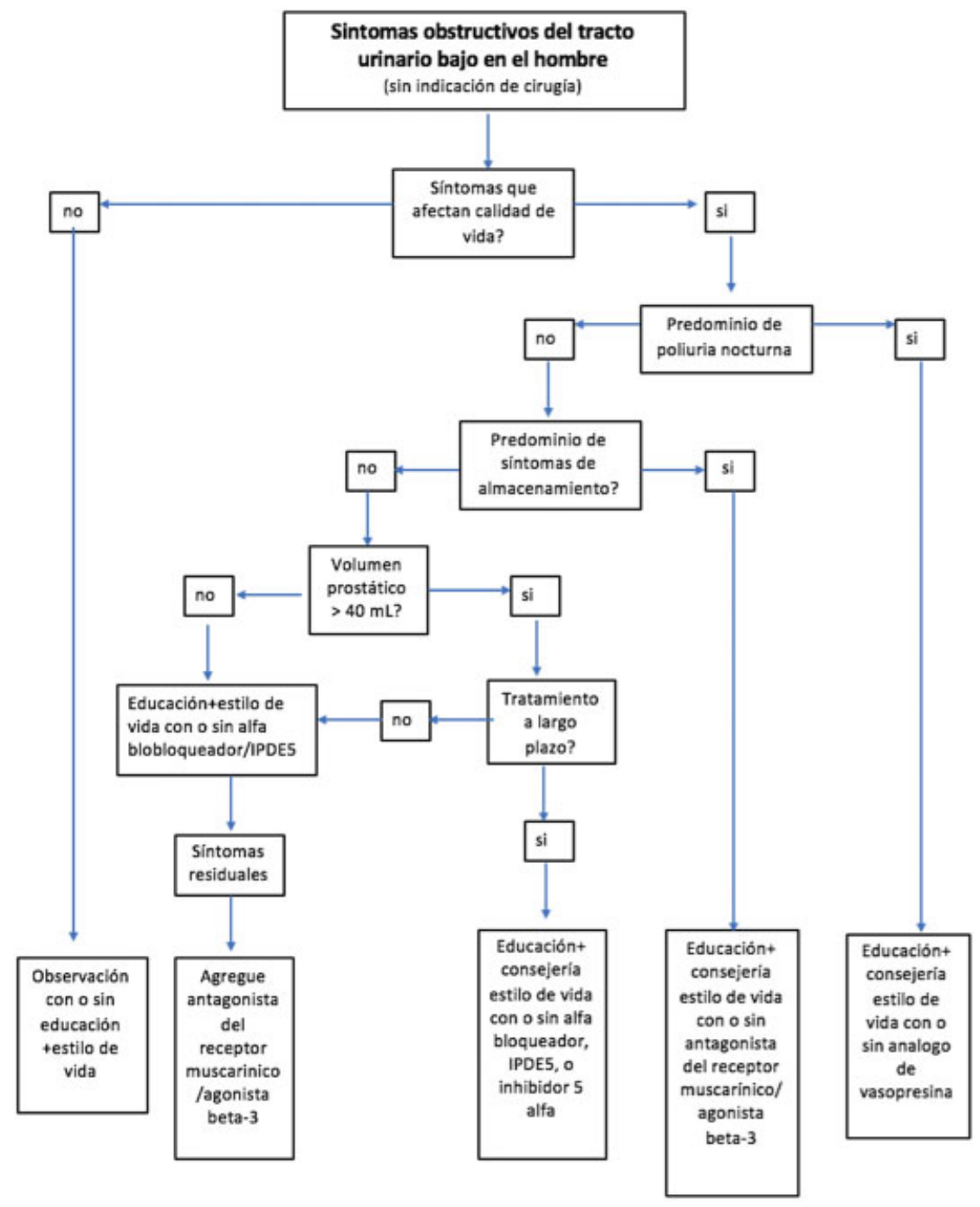

Flujograma 1 Algoritmo del tratamiento del hombre con SOUB empleando opciones de tratamiento médico y/o conservador. Las decisiones sobre el tratamiento dependen de los resultados encontrados durante las evaluaciones realizadas en la primera valoración. Note que las preferencias del paciente pueden modificar diferentes decisiones de tratamiento. 
mejoria similar en el Qmáx y el IPSS, justificado en estudios son de naturaleza retrospectiva. El Dispositivo Implantable Temporal en Nitinol) (DITN) es otra tecnología en investigación. Se están desarrollando estudios aleatorizados controlados comparando el DITN con un tratamiento de referencia. La ablación con agua guiada por imágenes con robot: AquaBeam en los estudios existentes no presentó resultados funcionales inferiores comparada con la RTUP en pacientes con SOUB y próstatas de $30-80 \mathrm{~mL}$. Es necesario el seguimiento a largo plazo para poder tener una evaluación clínica más asertiva de la Aqua-ablación. La ablación por energia de vapor de agua convectiva* (WAVE): El sistema Rezum requiere de estudios aleatorizados controlados contra una técnica de tratamiento de referencia para confirmar los resultados clínicos y para evaluar la eficacia y la seguridad a corto y mediano plazo del tratamiento con energía de vapor.

En cuanto a la embolización de arterias prostática, es mandatorio un estudio multidisciplinario que involucre urólogos y radiólogos, además de una adecuada selección de pacientes, que debe ser realizada por el urólogo y el radiólogo intervencionista. Los pacientes con próstatas de gran tamaño $(>80 \mathrm{~mL}$ ) son los que más se pueden beneficiar de la EAP. La escogencia de pacientes con SOUB que se beneficien de la EAP aún debe ser bien definida.

\subsection{Selección del paciente}

Los flujogramas presentados en el texto completo de la guía ilustran la selección del tratamiento de acuerdo al perfil del paciente y a la medicina basada en la evidencia.

\section{Nocturia}

Se deberá hacer una evaluación detallada de de la historia clínica de los pacientes con nocturia. No se ha realizado ningún estudio clínico de terapias dirigidas a la fisiopatología. La terapia antidiurética disminuye la frecuencia urinaria nocturna en hombres que tienen de base una severidad de síntomas mayor de dos episodios miccionales/noche, se ha asociado con sueño reparador. Nivel de evidencia $1 \mathrm{~b}$. Los medicamentos anticolinérgicos pueden disminuir la severidad de la urgencia urinaria nocturna, pero la reducción de la frecuencia es muy baja o no significativa. Las recomendaciones para ofrecer desmopresina a bajas dosis son débiles.

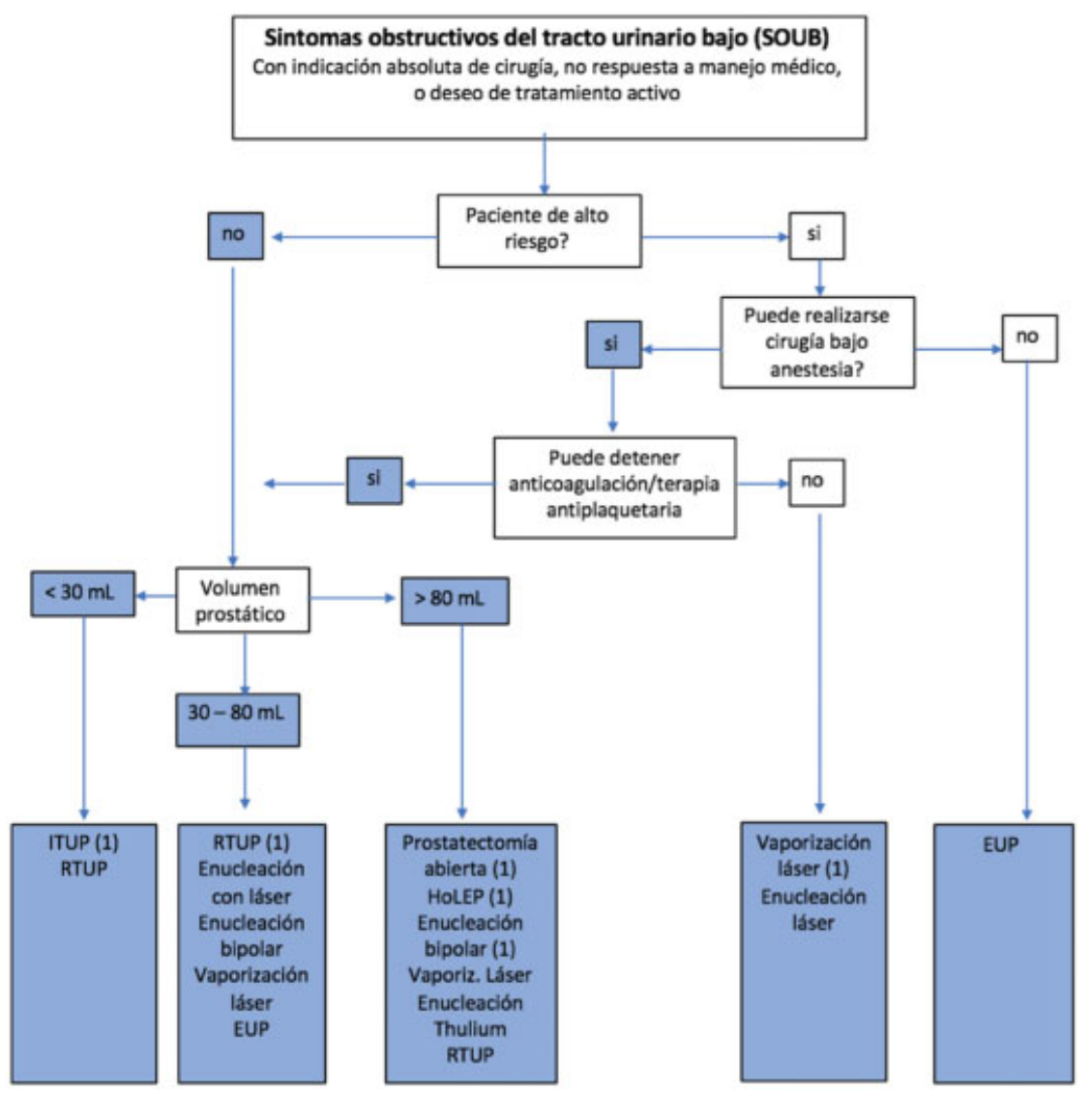

Flujograma 2 Algoritmo del tratamiento de los SOUB que afectan la calidad de vida y son refractarios al tratamiento conservador/médico o en los casos de indicación absoluta de cirugía. El diagrama de flujo está estratificado según el estado funcional del paciente para tolerar la anestesia, el riesgo cardiovascular y el tamaño de la próstata.

(1) Estándar actual/primera elección. Los tratamientos alternativos están listados en orden alfabético. La vaporización con láser incluye Greenlight, Thulium, y vaporización con láser de Diodo. La enucleación con láser incluye Holmium y Thulium.

HoLEP = Enucleación con láser holmium. ITUP = incision transuretral de la próstata. RTUP = prostatectomia transuretral y EUP = elevación de uretra prostática (Urolift). 


\section{Referencias}

1 NZGG. Handbook for the Preparation of Explicit Evidence-Based Clinical Practice Guidelines. New Zealand Guidelines Group; 2001:67-76

2 National Institute for Clinical Excellence (February 2004, updated 2005) Guideline Development Methods: Information for National Collaborating Centers and Guideline Developers. London: National Institute for Clinical Excellencehttp://www.nice.org.uk

3 Martin SA, Haren MT, Marshall VR, Lange K, Wittert GAMembers of the Florey Adelaide Male Ageing Study. Prevalence and factors associated with uncomplicated storage and voiding lower urinary tract symptoms in community-dwelling Australian men. World J Urol 2011;29(02):179-184https://www.ncbi.nlm.nih.gov/pubmed/ 20963421

4 d'Urologie SI(SIU), Lower Urinary Tract Symptoms (LUTS): An International Consultation on Male LUTS., C. Chapple \& P. Abrams, Editors. 2013https://www.siuurology.org/themes/web/assets/ files/ICUD/pdf/Male\%20Lower\%20Urinary\%20Tract\%20Symptoms $\% 20$ (LUTS).pdf

5 Kupelian V, Wei JT, O'Leary MP, et al; BACH Survery Investigators. Prevalence of lower urinary tract symptoms and effect on quality of life in a racially and ethnically diverse random sample: the Boston Area Community Health (BACH) Survey. Arch Intern Med 2006;166(21):2381-2387https://www.ncbi.nlm.nih.gov/pubmed/17130393

6 Agarwal A, Eryuzlu LN, Cartwright R, et al. What is the most bothersome lower urinary tract symptom? Individual- and population-level perspectives for both men and women. Eur Urol 2014; 65(06):1211-1217https://www.ncbi.nlm.nih.gov/pubmed/ 24486308

7 De Ridder D, Roumeguère T, Kaufman L. Urgency and other lower urinary tract symptoms in men aged $\geq 40$ years: a Belgian epidemiological survey using the ICIQ-MLUTS questionnaire. Int J Clin Pract 2015;69(03):358-365https://www.ncbi.nlm.nih.gov/pubmed/25648652

8 Kogan MI, Zachoval R, Ozyurt C, Schäfer T, Christensen N. Epidemiology and impact of urinary incontinence, overactive bladder, and other lower urinary tract symptoms: results of the EPIC survey in Russia, Czech Republic, and Turkey. Curr Med Res Opin 2014;30 (10):2119-2130https://www.ncbi.nlm.nih.gov/pubmed/ 24932562

9 Chapple CR, Wein AJ, Abrams P, et al. Lower urinary tract symptoms revisited: a broader clinical perspective. Eur Urol 2008;54(03):563-569https://www.ncbi.nlm.nih.gov/pubmed/ 18423969

10 Yang DY, Lee WK. A current perspective on post-micturition dribble in males. Investig Clin Urol 2019;60(03):142-147. Doi: 10.4111/icu.2019.60.3.142

11 Martin S, Lange K, Haren MT, Taylor AW, Wittert GMembers of the Florey Adelaide Male Ageing Study. Risk factors for progression or improvement of lower urinary tract symptoms in a prospective cohort of men. J Urol 2014;191(01): 130-137

12 Chapple CR, Al-Shukri SH, Gattegno B, et al. Tamsulosin oral controlled absorption system (OCAS) in patients with lower urinary tract symptoms suggestive of benign prostatic hyperplasia (LUTS/BPH): Efficacy and tolerability in a placebo and active comparator controlled phase 3a study. European Urology Supplements (ISSN 1569-9056) 2005;4:33-44

13 Karavitakis M, Kyriazis I, Omar MI, et al. Management of Urinary Retention in Patients with Benign Prostatic Obstruction: A Systematic Review and Meta-analysis. Eur Urol 2019;75(05):788-798. Doi: 10.1016/j.eururo.2019.01.046

14 Yuan J, Liu Y, Yang Z, Qin X, Yang K, Mao C. The efficacy and safety of alpha- 1 blockers for benign prostatic hyperplasia: an overview of 15 systematic reviews. Curr Med Res Opin 2013;29(03): 279-287
15 van Dijk MM, de la Rosette JJ, Michel MC. Effects of alpha(1)adrenoceptor antagonists on male sexual function. Drugs 2006; 66(03):287-301https://www.ncbi.nlm.nih.gov/pubmed/ 16526818

16 Nickel JC, Gilling P, Tammela TL, Morrill B, Wilson TH, Rittmaster RS. Comparison of dutasteride and finasteride for treating benign prostatic hyperplasia: the Enlarged Prostate International Comparator Study (EPICS). BJU Int 2011;108(03):388-394https:// www.ncbi.nlm.nih.gov/pubmed/21631695

17 Roehrborn CG. BPH progression: concept and key learning from MTOPS, ALTESS, COMBAT, and ALF-ONE. BJU Int 2008101 (Suppl 3):17-21https://www.ncbi.nlm.nih.gov/pubmed/18307681

18 Roehrborn CG, Abrams P, Rovner ES, Kaplan SA, Herschorn S, Guan Z. Efficacy and tolerability of tolterodine extended-release in men with overactive bladder and urgency urinary incontinence. BJU Int 2006;97(05):1003-1006https://www.ncbi.nlm.nih.gov/pubmed/ 16643482

19 Pattanaik S, Mavuduru RS, Panda A, et al. Phosphodiesterase inhibitors for lower urinary tract symptoms consistent with benign prostatic hyperplasia. Cochrane Database Syst Rev 2018;11:CD010060https://www.ncbi.nlm.nih.gov/pubmed/ 30480763

20 Buck AC. Is there a scientific basis for the therapeutic effects of serenoa repens in benign prostatic hyperplasia? Mechanisms of action. J Urol 2004;172(5 Pt 1):1792-1799https://www.ncbi.nlm. nih.gov/pubmed/15540722

21 Roehrborn CG, Oyarzabal Perez I, Roos EP, et al. Efficacy and safety of a fixed-dose combination of dutasteride and tamsulosin treatment (Duodart $(\circledR)$ ) compared with watchful waiting with initiation of tamsulosin therapy if symptoms do not improve, both provided with lifestyle advice, in the management of treatmentnaïve men with moderately symptomatic benign prostatic hyperplasia: 2-year CONDUCT study results. BJU Int 2015;116(03): 450-459https://www.ncbi.nlm.nih.gov/pubmed/25565364

22 Kaplan SA, Lee JY, Meehan AG, Kusek JW. Time Course of Incident Adverse Experiences Associated with Doxazosin, Finasteride and Combination Therapy in Men with Benign Prostatic Hyperplasia: The MTOPS Trial. J Urol 2016;195(06):1825-1829https://www. ncbi.nlm.nih.gov/pubmed/26678956

23 Drake MJ, Chapple C, Sokol R, et al; NEPTUNE Study Group. Longterm safety and efficacy of single-tablet combinations of solifenacin and tamsulosin oral controlled absorption system in men with storage and voiding lower urinary tract symptoms: results from the NEPTUNE Study and NEPTUNE II open-label extension. Eur Urol 2015;67(02):262-270https://www.ncbi.nlm.nih.gov/pubmed/25070148

24 Rassweiler J, Teber D, Kuntz R, Hofmann R. Complications of transurethral resection of the prostate (TURP)-incidence, management, and prevention. Eur Urol 2006;50(05):969-979, discussion 980https://www.ncbi.nlm.nih.gov/pubmed/16469429

25 Riedinger CB, Fantus RJ, Matulewicz RS, Werntz RP, Rodriguez JF, Smith ND. The impact of surgical duration on complications after transurethral resection of the prostate: an analysis of NSQIP data. Prostate Cancer Prostatic Dis 2019;22(02):303-308https://www. ncbi.nlm.nih.gov/pubmed/30385836

26 Mamoulakis C, Ubbink DT, de la Rosette JJ. Bipolar versus monopolar transurethral resection of the prostate: a systematic review and meta-analysis of randomized controlled trials. Eur Urol 2009; 56(05):798-809https://www.ncbi.nlm.nih.gov/pubmed/ 19595501

27 Omar MI, Lam TB, Alexander CE, et al. Systematic review and metaanalysis of the clinical effectiveness of bipolar compared with monopolar transurethral resection of the prostate (TURP). BJU Int 2014;113(01):24-35https://www.ncbi.nlm.nih.gov/pubmed/ 24053602

28 Inzunza G, et al. Bipolar or monopolar transurethral resection for benign prostatic hyperplasia? Reseccion transuretral bipolar o 
monopolar para hiperplasia prostatica benigna?. 2018;18: e7134https://www.ncbi.nlm.nih.gov/pubmed/29351269

29 Treharne C, et al. Economic Value of the Transurethral Resection in Saline System for Treatment of Benign Prostatic Hyperplasia in England and Wales: Systematic Review, Meta-analysis, and CostConsequence Model. Eur Urol focus 2016https://www.ncbi.nlm. nih.gov/pubmed/28753756

30 Autorino R, Damiano R, Di Lorenzo G, et al. Four-year outcome of a prospective randomised trial comparing bipolar plasmakinetic and monopolar transurethral resection of the prostate. Eur Urol 2009;55(04):922-929https://www.ncbi.nlm.nih.gov/pubmed/ 19185975

31 Poulakis V, Dahm P, Witzsch U, Sutton AJ, Becht E. Transurethral electrovaporization vs transurethral resection for symptomatic prostatic obstruction: a meta-analysis. BJU Int 2004;94(01):89-95https://www.ncbi.nlm.nih.gov/pubmed/ 15217438

32 Kaya C, Ilktac A, Gokmen E, Ozturk M, Karaman IM. The long-term results of transurethral vaporization of the prostate using plasmakinetic energy. BJU Int 2007;99(04):845-848https://www. ncbi.nlm.nih.gov/pubmed/17378844

33 Sun J, et al. Safety and feasibility study of holmium laser enucleation of the prostate (HOLEP) on patients receiving dual antiplatelet therapy (DAPT). World J Urol 2018;36;271https:// www.ncbi.nlm.nih.gov/pubmed/ 29138929

34 Briganti A, Naspro R, Gallina A, et al. Impact on sexual function of holmium laser enucleation versus transurethral resection of the prostate: results of a prospective, 2-center, randomized trial. J Urol 2006;175(05):1817-1821https://www.ncbi.nlm.nih.gov/pubmed/ 16600770

35 Thangasamy IA, Chalasani V, Bachmann A, Woo HH. Photoselective vaporisation of the prostate using $80-\mathrm{W}$ and $120-\mathrm{W}$ laser versus transurethral resection of the prostate for benign prostatic hyperplasia: a systematic review with meta-analysis from 2002 to 2012. Eur Urol 2012;62(02):315-323https://www.ncbi.nlm. nih.gov/pubmed/22575913

36 Świniarski PP, Stępień S, Dudzic W, Kęsy S, Blewniewski M, Różański W. Thulium laser enucleation of the prostate (TmLEP) vs. transurethral resection of the prostate (TURP): evaluation of early results. Cent European J Urol 2012;65(03):130-134https:// www.ncbi.nlm.nih.gov/pubmed/24578948

37 Becker B, Herrmann TRW, Gross AJ, Netsch C. Thulium vapoenucleation of the prostate versus holmium laser enucleation of the prostate for the treatment of large volume prostates: preliminary 6-month safety and efficacy results of a prospective randomized trial. World J Urol 2018;36(10): 1663-1671

38 Roehrborn CG, Barkin J, Gange SN, et al. Five year results of the prospective randomized controlled prostatic urethral L.I.F.T. study. Can J Urol 2017;24(03):8802-8813https://www.ncbi. nlm.nih.gov/pubmed/28646935

39 Sønksen J, Barber NJ, Speakman MJ, et al. Prospective, randomized, multinational study of prostatic urethral lift versus transurethral resection of the prostate: 12-month results from the BPH6 study. Eur Urol 2015;68(04):643-652https://www.ncbi. nlm.nih.gov/pubmed/25937539 